\title{
Frequency Tunable sub-THz Gyrotrons for Spectroscopy Applications
}

\author{
A. E. Fedotov ${ }^{1}, \underline{\text { I. V. Zotova }}{ }^{1}$, M. Yu. Glyavin ${ }^{1}$, R. M. Rozental ${ }^{1}$, A. S. Zuev ${ }^{1}$, \\ N. S. Ginzburg ${ }^{1}$, A. S. Sergeev ${ }^{1}$, and T. Idehara ${ }^{2}$ \\ ${ }^{1}$ Institute of Applied Physics of the Russian Academy of Sciences, Niznhny Novgorod, Russia, zotova@appl.sci-nnov.ru \\ ${ }^{2}$ Research Center for Development of Far-Infrared Region, University of Fukui, Japan
}

Development of methods of sub-THz mediumpower $(0.01$ to $1 \mathrm{~kW})$ radiation generation with wideband (several per cent) frequency tuning provides new possibilities for relevant fundamental and engineering applications. In particular, direct measurement of positronium hyperfine structure [1] would allow an experimental verification of a number of quantum electrodynamics predictions. On the other hand, $\mathrm{CW}$ tunable coherent radiation with specified parameters can be used in spectroscopy for detection of very smallintensity molecular spectra using the method of radioacoustic detection of absorption (RAD spectroscopy [2-4]). Most powerful and simultaneously most compact and accessible devices operating in the sub-THz range are the gyrotrons which have already confirmed their significance for a number of present-day issues. At the same time, one of gyrotron features is fairly narrow (less than percent) frequency tuning band which is a substantial limitation for gyrotron applications.

The main obstacle for implementation of a gyrotron with wide frequency tuning band is high Q-factor of the operating mode with one longitudinal variation near the cutoff frequency. Thus, for provision of smooth frequency tuning, excitation of higher longitudinal modes with relatively low Q-factor in the long resonators was usually used $[5,6]$. However, in this case, the part of Ohmic losses raise dramatically and, on the other hand, sensibility to the velocity spread in the electron beam increases. Both reasons lead to significant decrease in output power and strongly limit the frequency tuning.

In this paper, in contrast to most of the previous works, we propose using a relatively short cavity with low Q-factors to provide efficient excitation of a number of high-order axial modes. The main idea of the proposed method is exploiting the weaker sensitivity of a short-cavity gyrotron to the velocity spread in the electron beam. In fact, on a short resonator length the velocity spread does not have time for a significant influence on the electron-wave interaction process. At the same time, it is obvious that the shortening of the resonator will require increasing the current of the gyrotron electron beam. In addition to providing the starting conditions for exciting highorder longitudinal modes, it will also provide overlapping of the generation zones at neighboring modes. At the same time, the required current value can be substantially reduced by going to operation at low transverse modes due to the growth of the electronwave coupling coefficient. These considerations make it possible to expect that, with a certain optimization, such approaches can provide a sufficiently wide band of gyrotron generation.

Further using the KARAT code [7] we carried out PIC simulations for two gyrotron schemes. The first gyrotron with a frequency of about $200 \mathrm{GHz}$, a power of $0.5-1 \mathrm{~kW}$ and a tuning band of about $5 \%$ is intended for direct spectroscopy of positronium. The second gyrotron with a frequency of about $164 \mathrm{GHz}$, a power of $0.1-0.3 \mathrm{~kW}$ and a tuning band of about $3 \%$ is planned to be used for RAD spectroscopy experiments. The main parameters for both schemes are shown in Table 1.

Table 1. Parameters of tunable sub-THz gyrotrons

\begin{tabular}{|l|c|c|}
\hline Operating voltage & $20 \mathrm{kV}$ & $15 \mathrm{kV}$ \\
\hline Beam current & $1.5-2 \mathrm{~A}$ & $0.4 \mathrm{~A}$ \\
\hline Pitch-factor & \multicolumn{2}{|c|}{1.0} \\
\hline Operating mode & $\mathrm{TE}_{12}$ & $\mathrm{TE}_{02}$ \\
\hline Cavity radius & $1.25 \mathrm{~mm}$ & $2 \mathrm{~mm}$ \\
\hline Cavity length & $12-16 \mathrm{~mm}$ & $20 \mathrm{~mm}$ \\
\hline Beam injection radius & $0.72 \mathrm{~mm}$ & $0.55 \mathrm{~mm}$ \\
\hline Operating frequency & $\sim 204 \mathrm{GHz}$ & $164 \mathrm{GHz}$ \\
\hline Tuning band & $\sim 5 \%$ & $\sim 3 \%$ \\
\hline Output power & $0.5-1 \mathrm{~kW}$ & $0.1-0.3 \mathrm{~kW}$ \\
\hline
\end{tabular}

Further results of PIC-simulations are presented in detail for the case of $200 \mathrm{GHz}$ gyrotron. The geometry of interaction space and instantaneous positions of charged macroparticles are shown in Fig.1a,b. The simulations evidence that for the velocity spread of $20 \%$ the radiation power exceeds $0.5 \mathrm{~kW}$ in the entire frequency tuning band of $10 \mathrm{GHz}(5 \%)$ (see curve 1 in Fig.2). For this region of the magnetic field, from 7.3 to $8.6 \mathrm{~T}$, stationary single-mode excitation of the operating $\mathrm{TE}_{12}$ mode takes place (Fig. 1c). For a magnetic field higher than $8.6 \mathrm{~T}$, the excitation of the parasitic transverse $\mathrm{TE}_{51}$ mode occurs, which limits the further increase in the bandwidth. The use of the proposed gyrotron operation regime with a short cavity and a high electron current ensure a wide frequency tuning band even for a very high spread, $50 \%$. This result confirms the small influence of the velocity spread on the electron-wave interaction for shortcavity gyrotron. 


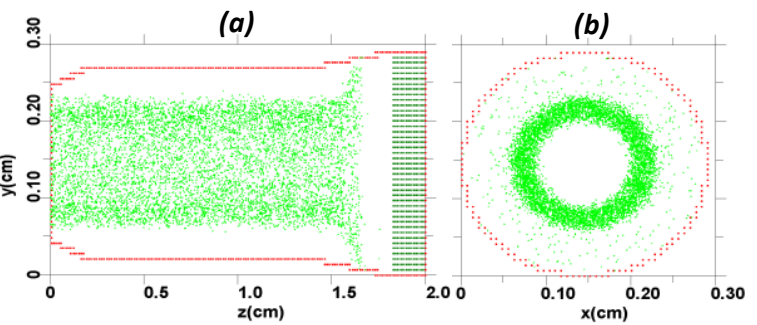

(c)
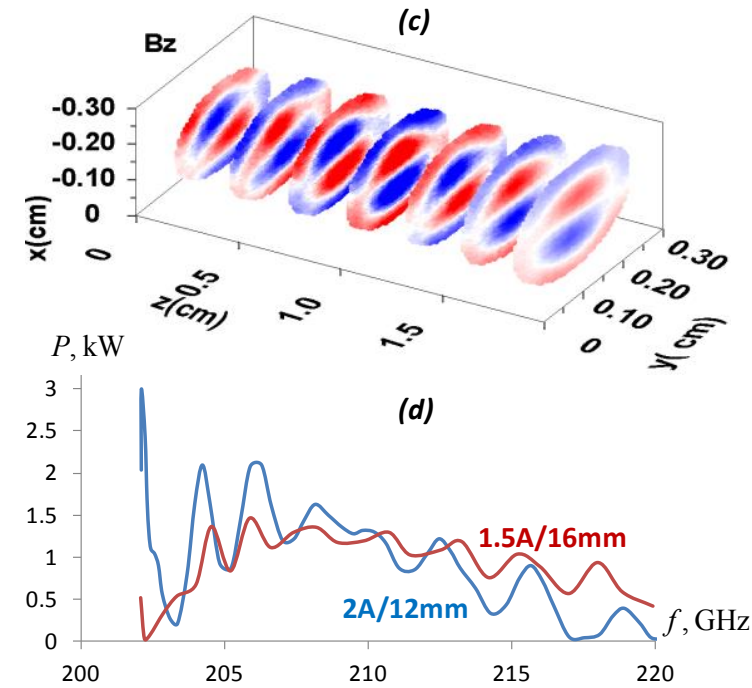

Fig.1. (a, b) Geometry of the gyrotron cavity used in 3D PIC simulations of $200 \mathrm{GHz}$ gyrotron. (c) The transverse structure of the excited $\mathrm{TE}_{12}$ mode. (d) The gyrotron output power vs. radiation frequency for different cavity lengthes and electron currents

It should be noted that for the further improvement of the output characteristics of a tunable gyrotron, methods for reducing ohmic losses and eliminating gaps on the power vs frequency curve should be employed. These possible methods, which are the subjects of the further research, include a smooth taper at the output end of the cavity $[6,8]$, a cavity profile taper [9], and a cathode-end power output configuration of the device $[10,11]$.

The work was supported by the Russian Science Foundation under grant No. 18-12-00394.

\section{References}

1. Yamazaki T., Miyazaki A., Suehara T., Namba T., Asai S., Kobayashi T., Saito H., Ogawa I., Idehara T., Sabchevski S. Direct Observation of the Hyperfine Transition of Ground-State Positronium // Phys. Rev. Lett. 2012. V. 108. No. 25. Art.no. 253401.

2. Belov S.P., Kazakov V.P., Krupnov A.F., Markov V.N., Mel'nikov A.A., Skvortsov V.A., Tret'yakov M.Yu. The study of microwave pressure lineshifts // J. Mol. Spectros. 1982. V.94. No. 2. P. 264-282.

3. Li H., Le Roy R.J. Quadrupole moment function and absolute infrared quadrupolar intensities for $\mathrm{N}_{2} / / \mathrm{J}$. Chem. Phys. 2007. V. 126. No. 22. Art.no. 224301.

4. Ba Y.A, Wenger C., Surleau R., Boudon V., Rotger M., Daumont L., Bonhommeau D.A., Tyuterev V.G., Dubernet M.-L. MeCaSDa and ECaSDa: Methane and ethene calculated spectroscopic databases for the virtual atomic and molecular data centre // J. Quant. Spectrosc. Radiat. Transf. 2013. V.130. P.62-68.

5. Chang T.H., Idehara T., Ogawa I., Agusu L., and Kobayashi S. Frequency tunable gyrotron using backwardwave components // J. Appl. Phys. 2009. V. 105. No. 6. Art. no. 063304 .

6. Barnes A.B., Nanni E.A., Hertzfeld J., Griffin R.G., and Temkin R.J. A $250 \mathrm{GHz}$ gyrotron with a $3 \mathrm{GHz}$ tuning bandwidth for dynamic nuclear polarization // J. Magn. Resonance. 2012. V. 221. P. 147-153.

7. Tarakanov V.P. Code KARAT in simulations of power microwave sources including Cherenkov plasma devices, vircators, orotron, E-field sensor, calorimeter etc. // EPJ Web of Conferences. 2017. V. 149. Art.no. 04024.

8. Torrezan A.C., Shapiro M.A., Sirigiri J.R., Temkin R.J., Griffin R.G. Operation of a Continuously FrequencyTunable Second-Harmonic CW 330-GHz Gyrotron for Dynamic Nuclear Polarization // IEEE Trans. Electron. Dev. 2011. V. 58. No. 8. P. 2777-2783.

9. Qi X.-B., Du C.-H., Pan S., Ji X., Huang B., Liu P.$K$. Terahertz Broadband-Tunable Minigyrotron With a Pulse Magnet // IEEE Trans. Electron. Dev. 2017. V.64. No. 2. P. 527-535.

10. Chang T.H., Chen S.H. Stepwise frequency tuning of a gyrotron backward-wave oscillator // Phys. Plasmas. 2004. V. 12. No. 1. Art.no. 013104.

11. Bratman V.L., Fedotov A.E., Kalynov Yu.K., Osharin I.V., Zavolsky N.A. Smooth Wideband Frequency Tuning in Low-Voltage Gyrotron With Cathode-End Power Output // IEEE Trans. Electron Dev. 2017. V. 64. No. 12. P. 5147-5150. 